\title{
Characterization of Refractory Organic Matter in Pulp and Paper Mill Effluents
}

\author{
Masayuki Watanabe ${ }^{* 2, a)}$, Keiko Fujita, \\ Hiro Iwata, Yutaka Nuruki, Kazuko Watanabe, \\ Miyuki Nakagawa and Hitoshi Okada \\ Japan Pulp and Paper Research Institute, Inc. ${ }^{* 1}$
}

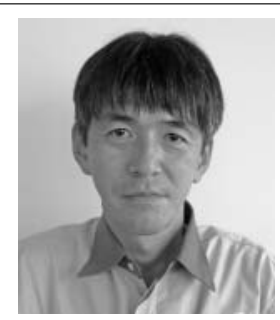

Masayuki Watanabe

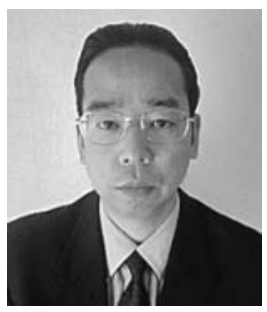

Yutaka Nuruki

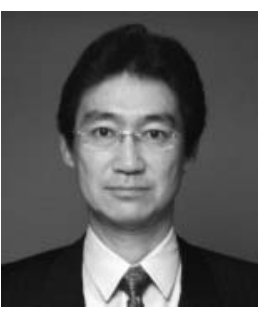

Hitoshi Okada

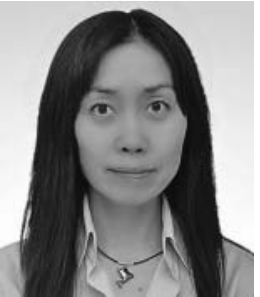

Keiko Fujita

Kazuko Watanabe

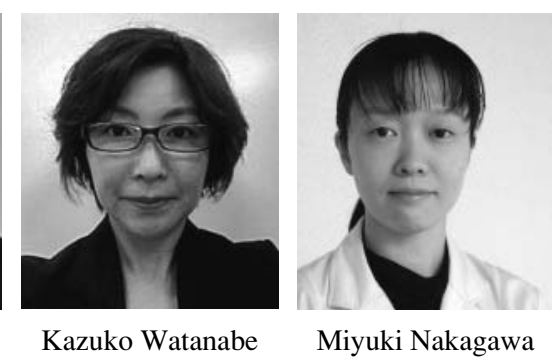

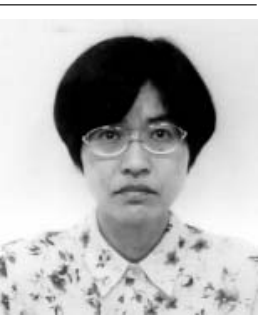

Hiro Iwata

Miyuki Nakagawa

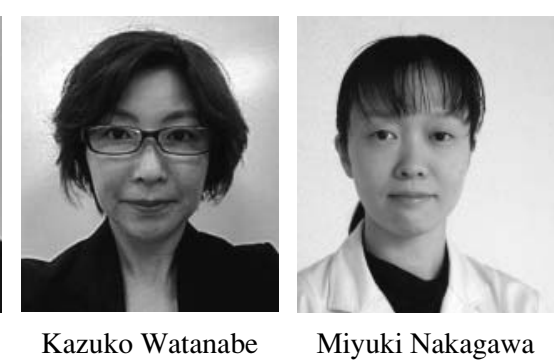

\begin{abstract}
The quality of wastewater from Japanese pulp and paper mills has been recently improved, due to regulation of effluent standards which has been stricter since the Water Pollution Control Law was enforced in 1972. However, it has not attained yet in some mills that the level of COD (Chemical Oxygen Demand) of the wastewater becomes below the environmental standard. Accumulation of refractory organic matter has been pointed out as a new problem especially for a part of the closed water area in spite of regulation of total emission. Japan Pulp and Paper Research Institute is researching this problem.

As the first step of this theme, the characterization of the refractory organic matter was studied. Dissolved organic matter in wastewater was fractionated into five fractions ; hydrophobic acid, hydrophobic neutral, base, hydrophilic acid, hydrophilic neutral. Based on measurements of the degradation rates of the fractionated compo-
\end{abstract}

\footnotetext{
${ }^{* 1}$ 5-13-11 Tokodai, Tsukuba-shi, Ibaraki 300-2635, Japan

${ }^{* 2} \mathrm{E}-$ mail : 0940124@nipponpapergroup.com

${ }^{a)}$ Current address : NPi Research Laboratory, Nippon Paper Industries Co., Ltd./5-21-1 Oji, Kita-ku, Tokyo 114-0002, Japan
} 
nents, it was confirmed that the most refractory matter was fractionated into the hydrophobic acid.

Furthermore, we compared softwood and hardwood kraft pulp (KP) bleaching drainages. Softwood KP bleaching drainage was more resistant to decomposition than hardwood KP bleaching drainage, and contained the hydrophobic acid in a higher ratio. In addition, the hydrophobic acid in softwood in hardwood KP bleaching drainage.

In order to investigate the source of the hydrophobic acid, the each process drainage was fractionated as well. As a result, the maximum source of the hydrophobic acid was KP bleaching process. Pyrolysis GC-MS analysis of the hydrophobic acid of bleaching drainage indicated that lignin was a major composition of the refractory organic matter.

Keywords : refractory organic matter, hydrophobic acid

\section{Introduction}

Environmental regulations including the Water Pollution Control Act enacted in 1972 have significantly improved water environment in Japan by reducing effluent pollutants. In several parts of semi-enclosed sea, however, the environmental standard for chemical oxygen demand (COD) has not been attained yet. The COD reduction of lakes and marshes is unsatisfactory and in several areas, COD has been gradually increasing. It is likely that the accumulation of recalcitrant organic substances such as humic acids that are hard-biodegradable by natural microorganisms accounts for most of the COD increase ${ }^{1,2,3)}$.

Abatement of refractory organics in mill effluents presents a serious challenge for the pulp and paper industries because they consume much volume of water, taking the environmental protection into consideration ${ }^{4,5,6)}$. In such a situation, the rational first step was to characterize refractory organics.

As pulp and paper mill effluents contain various organic chemicals, it is difficult to evaluate characteristics of each component. Imai et al. of National Institute of Environmental Studies ${ }^{1)}$ fractionated dissolved organic matter (DOM) in water from a lake, inflowing rivers, and several other DOM sources using resin adsorbents into five classes: aquatic humic substances (AHS), hydrophobic neutrals $(\mathrm{HoN})$, hydrophilic acids (HiA), bases $(\mathrm{BaS})$, and hydrophilic neutrals $(\mathrm{HiN})^{2,3)}$. They used Total Organic Carbon (TOC) as an index of total organic substances instead of COD, eliminating issues associated with COD measurements such as use of weak oxidizing chemical, poor reproducibility of measurement data and effects of reductive inorganics.

The objective of this study was to apply the DOM fractionation method to pulp and paper mill effluents and to find the most refractory fractions and their origins from which they have derived.

\section{Materials and Methods}

\section{1 Collection of effluent samples}

Effluent samples were collected from a final outflow to the environment and process streams in several pulp and paper mills owned by Oji Holdings Corporation and Nippon Paper Industries Co., Ltd. respectively.

Major components in pulp mill effluents consist of methanol, organic acids, carbohydrates and lignin. As reference chemicals, formic acid, acetic acid, cellobiose, xylooligosaccharide and alkali lignin were selected for the DOM fractionation.

\subsection{Biodegradation test}

$100 \mathrm{ml}$ of activated sludge in a mill plant was centrifuged at 2,000 rpm, for 5 minutes. The clear top layer of the sludge was removed and the bottom layer was mixed with bleach plant effluent to make $100 \mathrm{ml}$ sample. The sample was treated with biological contact aeration at 25 degrees Celsius for 24 hours. The biologically treated sample was then centrifuged at 2,000 rpm, for 5 minutes. The clear top layer of the sample was collected and used for the DOM fractionation. The same tests were performed on other samples.

\subsection{DOM fractionation}

Samples were fractionated by using ion exchange resins into five fractions : hydrophobic acids, hydrophobic neutrals, bases, hydrophilic acids and hydrophilic neutrals. A flow schematic of the DOM fractionation procedure is shown in Fig. 1. The steps were as follows:

I . The non-ionic resin column was rinsed 3 times, alternating hyperpure water, $\mathrm{NaOH}$ and $\mathrm{HCl}$ before application of samples. A blank sample (B 1) was collected from the final rinse with $\mathrm{HCl}$.

II. Cationic resin and anionic resin were packed into two glass columns separately. Glass columns containing the cationic resin and the anionic resin were connected in series and conditioned by pumping hyperpure water through the resins. Blank samples (B 2 and B 3) were collected from each column after condi- 


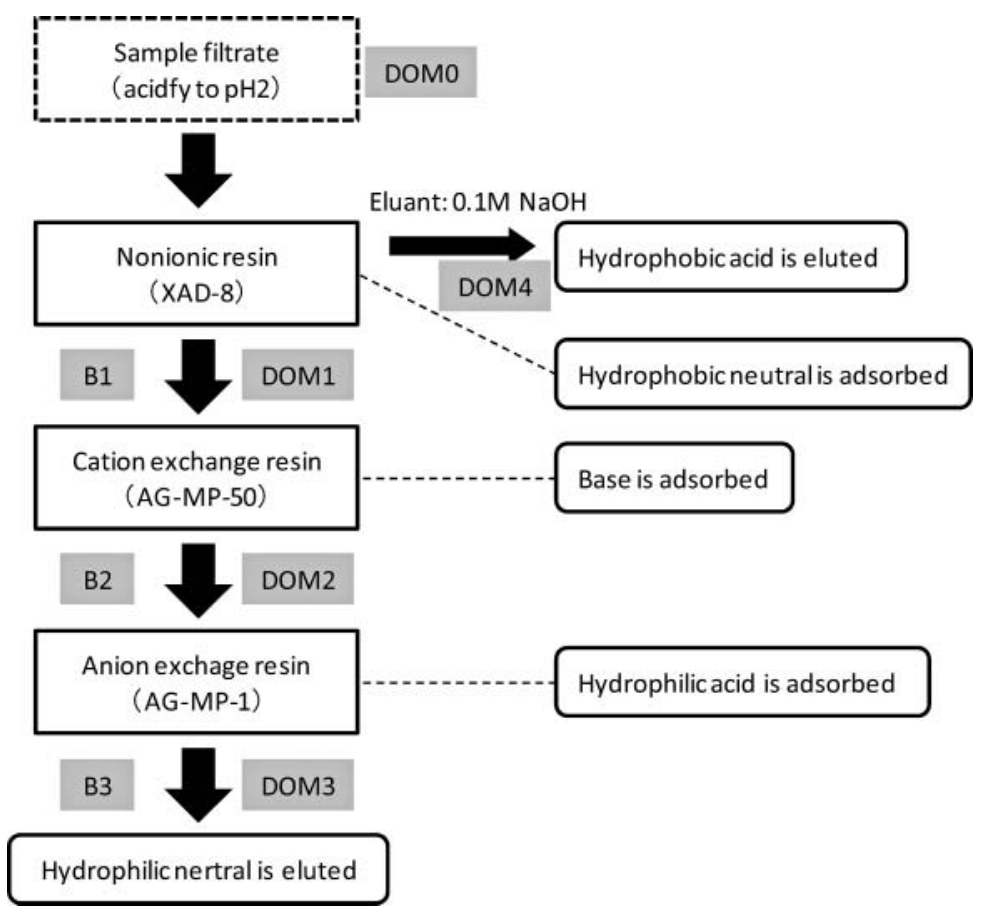

Fig. 1 Schematic diagram of the procedure for fractionation

tioning:

III. Pass the sample through the glass filter $(\mathrm{GF} / \mathrm{F}, 1$ $\mu \mathrm{m})$ and acidify the filtrate to $\mathrm{pH} 2.0$ with $\mathrm{HCl}$ (DOM $0)$.

IV . Pass the sample through the non-ionic resin column and rinse the column with $\mathrm{HCl}$.

$\mathrm{V}$. Elute the column in the reverse direction with $\mathrm{NaOH}$ (DOM 4).

VI. Pass the sample through the series of cation-anion resin columns and collect eluant samples from the cation resin column (DOM 2) and then from the anion resin column (DOM 3).

After fractionation, dissolved organic carbon (DOC) measurement was conducted with a Shimadzu TOCVCPH total-organic carbon analyzer for blank samples B 1-B 3 and DOMs 0-4. Each DOM fraction was calculated as follows :

Hydrophobic acids $(\mathrm{mg} / \mathrm{l})=$ DOM $4 \quad \cdots \cdot \cdot(1)$

Hydrophobic neutrals $(\mathrm{mg} / \mathrm{l})=$ DOM $0-$ DOM $4-$

$$
\text { (DOM 1-B 1) } \cdots \cdot \cdot \cdot(2)
$$

$\operatorname{Bases}(\mathrm{mg} / \mathrm{l})=(\mathrm{DOM} 1-\mathrm{B} 1)-(\mathrm{DOM} 2-\mathrm{B} 2) \cdots \cdots \cdot(3)$

Hydrophilic acids $(\mathrm{mg} / \mathrm{l})=(\mathrm{DOM} 2-\mathrm{B} 2)-$

$$
\text { (DOM } 3-\mathrm{B} 3) \quad \cdots \cdot(4)
$$

Hydrophilic neutrals $(\mathrm{mg} / \mathrm{l})=$ DOM $3-$ B $3 \quad \cdots \cdot(5)$

\subsection{Analytical method}

In order to characterize hydrophobic acids and hydrophilic acids, freeze-dried DOM fractions were measured by pyrolysis gas chromatography and mass spectrometry. Measurement was commissioned to Nippon Paper Industries. Analytical conditions were as follows :

- Pyrolysis temperature : $500^{\circ} \mathrm{C}$
- GC column : DB-1 $30 \mathrm{~m} \times 0.25 \mathrm{~mm} \times 1 \mu \mathrm{m}$

- Temperature profile : $50^{\circ} \mathrm{C}$-(increase $5{ }^{\circ} \mathrm{C} /$ minute)$300^{\circ} \mathrm{C}$ (hold $300^{\circ} \mathrm{C}$ for 10 minute)

- Split ratio : $1 / 60$

\section{Results and Discussion}

\subsection{Final effluent discharge}

Dissolved organic matter (DOM) fractionation of effluents from 26 pulp and paper mills was shown in Fig. 2. Samples were collected from final combined effluents discharged to the environment. Samples prior to biodegradation treatment exhibited DOC variations in the range of 20 $\mathrm{mg} / \mathrm{l}$ to $70 \mathrm{mg} / \mathrm{l}$ depending on pulping process, pulp production and effluent treatment. After biodegradation treatment, DOC variations dropped to the range of $6 \mathrm{mg} / \mathrm{l}$ to $24 \mathrm{mg} / 1$.

DOM-fraction distribution patterns were significantly different depending on the origin of the samples as is shown in Table 1. Prior to biodegradation treatment, hydrophilic acids fraction (53\%) and hydrophobic acids fraction $(24 \%)$ were found to be the dominant fractions. DOM -fraction distribution patterns changed drastically after biodegradation treatment. Hydrophobic acids fraction increased from $24 \%$ to $52 \%$ and hydrophilic acids fraction decreased from $53 \%$ to $34 \%$. Biodegradation ratio of hydrophobic acids fraction was the lowest. It was $32 \%$ while other DOM-fractions were in the range of $75 \%$ to $89 \%$. Hydrophobic acids fraction contains recalcitrant organics.

\section{2 Process streams}

From the findings ${ }^{8)}$ that COD reduction rate of bleach plant effluent by active biological sludge treatment was 


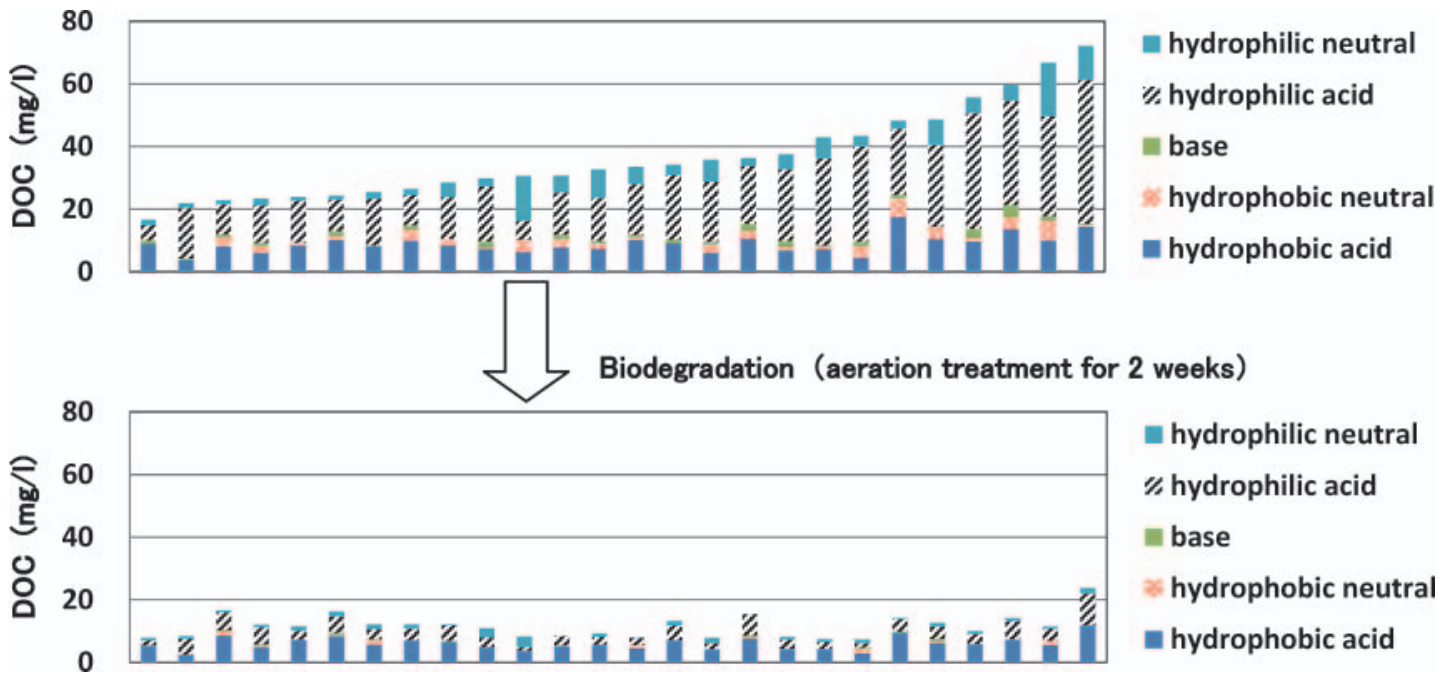

Fig. 2 Fractionation of dissolved organic matter (DOM) of effluents from 26 pulp and paper mills Legend : DOC : Dissolved Organic Carbon

Table 1 Average DOC, component ratio, and biodegradation rate of each fraction in effluents from 26 pulp and paper mills

\begin{tabular}{|c|c|c|c|c|c|}
\hline & \multicolumn{2}{|c|}{ Before biodegradation } & \multicolumn{2}{|c|}{ After biodegradation } & \multirow{2}{*}{$\begin{array}{l}\text { Biodegradation } \\
\text { rate }(\%)\end{array}$} \\
\hline & $\mathrm{DOC}(\mathrm{mg} / \mathrm{l})$ & $\begin{array}{l}\text { Component } \\
\text { ratio }(\%)\end{array}$ & $\mathrm{DOC}(\mathrm{mg} / \mathrm{l})$ & $\begin{array}{l}\text { Component } \\
\text { ratio (\%) }\end{array}$ & \\
\hline Hydrophobic acid & 8.7 & 24 & 6.0 & 52 & 32 \\
\hline Hydrophobic neutral & 2.2 & 6 & 0.3 & 3 & 87 \\
\hline Base & 1.1 & 3 & 0.2 & 2 & 89 \\
\hline Hydrophilic acid & 19.6 & 53 & 3.9 & 34 & 76 \\
\hline Hydrophilic neutral & 5.0 & 14 & 1.1 & 9 & 75 \\
\hline
\end{tabular}

smaller than that of other process effluents, it was estimated that bleach plant effluent contains refractory organics. A final combined effluent discharged to the environment consists of kraft pulp black liquor evaporator drain, paper machine white water and bleach plant effluent. DOC and DOM-fraction distribution patterns of these three streams were shown in Fig. 3. Hydrophilic neutrals (86\%) were found to be the dominant fraction in DOM fractions in evaporation drain. Hydrophilic acids (60\%) and hydrophobic acids (30\%) were dominant in bleach effluent. Hydrophilic acids were $76 \%$ of DOM fractions in paper machine white water.

From the previous findings ${ }^{9)}$ it was estimated that the prime component of hydrophilic neutrals in evaporation drain was methanol. The prime components of hydrophilic acids in bleach effluent were organic acids and carbohydrates. And the prime component of hydrophobic acids in bleach effluent was lignin. Lignin is refractory organic ${ }^{10)}$. Fig. 3 shows that the origin of hydrophobic acids was a bleach plant.
Characteristics of bleach plant effluents from softwood pulp and hardwood pulp were shown in Table 2. Total amount of hydrophobic and hydrophilic acids fractions were about the same in both effluents. They were approximately $90 \%$. But the ratio of hydrophobic acids in DOM fractions were different : softwood was $49 \%$ while hardwood was $30 \%$. After bio-degradation treatment for 24 hours by using active biological sludge of mill facilities, hydrophobic acids fraction dropped by $12 \%$ in softwood and by $47 \%$ in hardwood. It was reported ${ }^{10)}$ that softwood contains more lignin than hardwood does, and softwood lignin is more refractory than hardwood lignin. From Table 2, it was estimated that hydrophobic acids fraction in bleach effluent was lignin.

\subsection{Reference chemicals}

In order to confirm our estimation mentioned above ${ }^{9)}$, the DOM fractionation experiment was performed for reference chemicals : formic acid, acetic acid, cellobiose, xylooligosaccharide and alkali lignin. The results were shown in Table 3. 99\% of methanol was classified as hydrophilic 


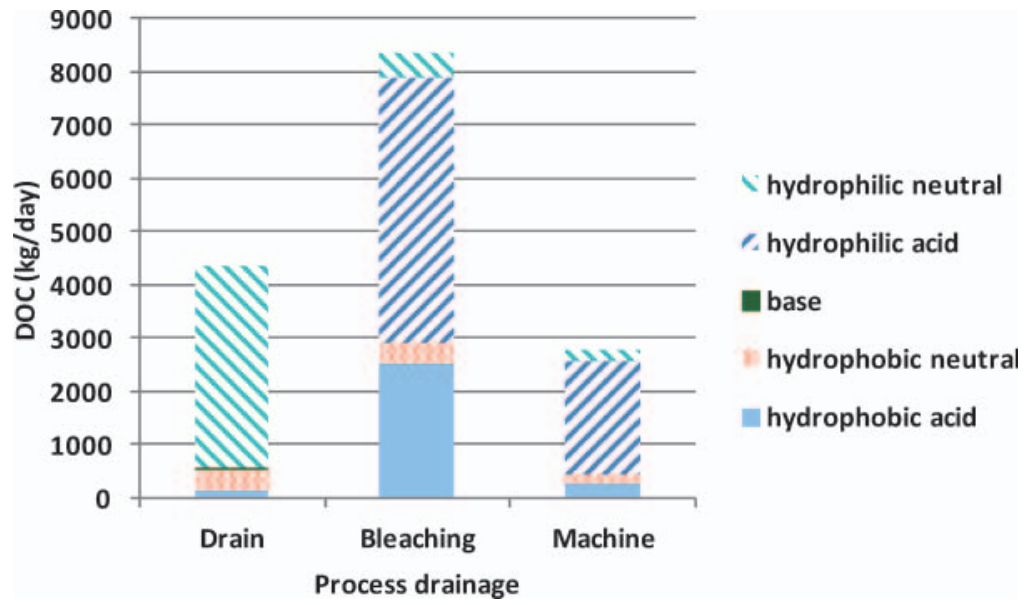

Fig. 3 Fractionation of quantity of DOM generation in each process drainage of the BKP mill

Table 2 Comparison of DOM fractions between hardwood and softwood pulp bleachings

\begin{tabular}{l|c|c|c|c}
\hline \multirow{2}{*}{} & \multicolumn{2}{|c|}{ Component ratio (\%) } & \multicolumn{2}{c}{ Biodegradation ratio (\%) } \\
\cline { 2 - 5 } & Hardwood & Softwood & Hardwood & Softwood \\
\hline Hydrophobic acid & 30 & 49 & 47 & 12 \\
\hline Hydrophobic neutral & 0 & 8 & - & - \\
\hline Base & 1 & 1 & - & - \\
\hline Hydrophilic acid & 63 & 36 & 79 & - \\
\hline Hydrophilic neutral & 6 & 5 & - & - \\
\hline
\end{tabular}

※iodegradation : Activated sludge treatment for $24 \mathrm{hrs}$

Table 3 Fractionation of various compounds related to wood components

\begin{tabular}{l|c|c|c|c|c|c}
\hline & \multicolumn{6}{|c}{ Component ratio (\%) } \\
\cline { 2 - 7 } & Methanol & Acetic acid & Formic acid & Cellobiose & $\begin{array}{c}\text { Xylo } \\
\text { origosaccharide }\end{array}$ & $\begin{array}{c}\text { Alkali } \\
\text { lignin }\end{array}$ \\
\hline Hydrophobic acid & 1 & 1 & 1 & 1 & 1 & 32 \\
\hline Hydrophobic neutral & 0 & 2 & 2 & 0 & 0 & 12 \\
\hline Base & 0 & 0 & 0 & 0 & 0 & 8 \\
\hline Hydrophilic acid & 0 & 96 & 95 & 97 & 98 & 11 \\
\hline Hydrophilic neutral & 99 & 1 & 2 & 2 & 1 & 37 \\
\hline
\end{tabular}

neutrals. $96 \%$ of acetic acids and $95 \%$ of formic acids were hydrophilic acids. Refractory alkali lignin consisted of $37 \%$ hydrophilic neutrals and $32 \%$ hydrophobic acids. Thus the estimation" ${ }^{9)}$ was verified : the prime component of hydrophobic acids fraction was lignin.

\section{4 Prime components in bleach effluent}

$60 \%$ hydrophilic acids fractions and $30 \%$ hydrophobic acids in bleach effluent were analyzed with pyrolysis gas chromatography. Their pyrograms were shown in Fig. 4. Peaks of furfural, methyl furfural and furfuryl alcohol were detected in hydrophilic acids. Thus carbohydrates were confirmed to be the prime component of hydrophilic acids. Peaks of phenol, guaiacol, vinyl guaiacol, syringol, vanillin and homovanillic acid were found in hydrophobic acids. It was confirmed that lignin was the prime component of hydrophobic acids.

\section{Conclusion}

Abatement of refractory organics in mill effluents presents a serious challenge for the pulp and paper industries. 


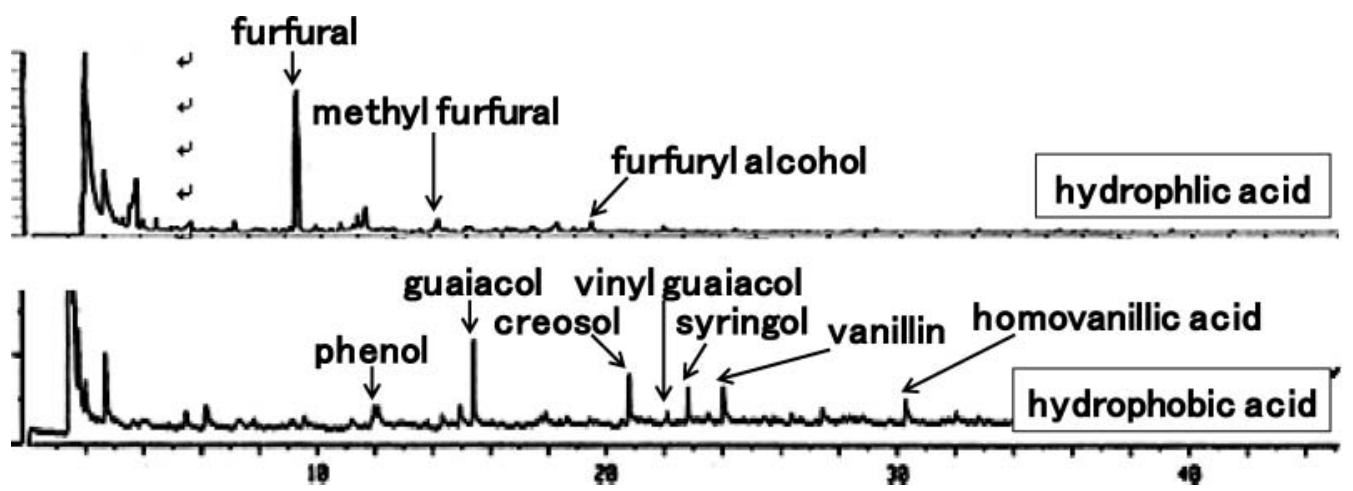

Fig. 4 Pyrograms of hydrophilic acid and hydrophobic acid fractions

The first step of our study was to characterize recalcitrant organics. As pulp and paper mill effluents contain various organic chemicals, it is difficult to evaluate characteristics of each component. Samples were fractionated by using ion exchange resins into five fractions : hydrophobic acids, hydrophobic neutrals, bases, hydrophilic acids and hydrophilic neutrals. Hard-biodegradable organics were found in hydrophobic acids fraction.

In order to find the origin of hydrophobic acids fraction, several effluent streams in pulp and paper mills were analyzed. It was found that much hydrophobic acids were detected in softwood bleach plant effluent than in hardwood bleach plant effluent. They were difficult to be degraded by biological treatment. Its prime component in hydrophobic acids was organic compounds derived from lignin.

The DOM fractionation method was successfully applied to characterize refractory organics in pulp and paper mill effluents. The future research was needed to control and reduce hydrophobic acids fractions in bleach plant effluents.

\section{References}

1) Akio Imai, Takehiko Fukushima, Kazuo Matsushige, and Yong Hwan Kim : Water Research 35 (17) 4019 -4028 (2001)

2) National Institute for Environmental Studies, Japan, Special Research Report：SR-36-2001 (2001)

3) National Institute for Environmental Studies, Japan, Special Research Report: SR-62-2004 (2004)

4) Takagi H. : JAPAN TAPPI J.61 (7) 812-816 (2007)

5) Takagi H. : JAPAN TAPPI J.63 (4) 378-382 (2009)

6) Takagi H., et al. : JAPAN TAPPI J.63 (9) 1091-1104 (2009)

7) Iwata H. : JAPAN TAPPI J. 63 (10) 1225-1237 (2009)

8) Ueda M. : JAPAN TAPPI J. 58 (10) 1373-1378 (2004)

9) Pulp and Papermaking Science and Technology, vol. 10, Environmental control, JAPAN TAPPI Press, Tokyo, 1966, p 103-108

10) Wood Chemistry, Buneido Publishing Co., Ltd., 2010, p 277-327

(Manuscript accepted 24 May, 2013) 